\title{
Pleolipoviridae, a newly proposed family comprising archaeal pleomorphic viruses with single-stranded or double-stranded DNA genomes
}

\author{
Maija K. Pietilä ${ }^{1} \cdot$ Elina Roine $^{2} \cdot$ Ana Sencilo $^{3}$ - Dennis H. Bamford ${ }^{2}$. \\ Hanna M. Oksanen ${ }^{2}$
}

Received: 17 June 2015/ Accepted: 13 September 2015/Published online: 12 October 2015

(C) Springer-Verlag Wien 2015

\begin{abstract}
Viruses infecting archaea show a variety of virion morphotypes, and they are currently classified into more than ten viral families or corresponding groups. A pleomorphic virus morphotype is very common among haloarchaeal viruses, and to date, several such viruses have been isolated. Here, we propose the classification of eight such viruses and formation of a new family, Pleolipoviridae (from the Greek pleo for more or many and lipos for lipid), containing three genera, Alpha-, Beta-, and Gammapleolipovirus. The proposal is currently under review by the International Committee on Taxonomy of Viruses (ICTV). The members of the proposed family Pleolipoviridae infect halophilic archaea and are nonlytic. They share structural and genomic features and differ from any other classified virus. The virion of pleolipoviruses is composed of a pleomorphic membrane vesicle enclosing the genome. All pleolipoviruses have two major structural
\end{abstract}

This article is related to an ongoing taxonomic proposal, submitted to the ICTV but not yet accepted at the time of submission. The taxonomy proposed here has not been endorsed by the ICTV Executive Committee, may differ from any new taxonomy that is ultimately approved by the ICTV, and is presented for discussion only but has no official standing.

Hanna M. Oksanen

hanna.oksanen@helsinki.fi

1 Department of Food and Environmental Sciences, University of Helsinki, P.O. Box 56, Viikinkaari 9, 00014 Helsinki, Finland

2 Department of Biosciences and Institute of Biotechnology, University of Helsinki, P.O. Box 56, Viikinkaari 9, 00014 Helsinki, Finland

3 Laboratory of Molecular Biology of Bacterial Pathogens, Institute of Microbiology of the ASCR, v.v.i., Czech Academy of Sciences, 14220 Prague 4, Czech Republic protein species, internal membrane and spike proteins. Although the genomes of the pleolipoviruses are single- or double-stranded, linear or circular DNA molecules, they share the same genome organization and gene synteny and show significant similarity at the amino acid level. The canonical features common to all members of the proposed family Pleolipoviridae show that they are closely related and thus form a new viral family.

\section{Introduction}

Archaea and their viruses thrive in extreme environments, and most of archaeal viruses characterized so far infect extremophiles, either hyperhalophiles or hyperthermophiles [4-6, 29]. Viruses infecting archaea display diverse virion morphotypes, some of which are unique. Consequently, archaeal viruses have been classified into over ten viral families and one floating genus, Salterprovirus, by the International Committee on Taxonomy of Viruses (ICTV) [19, 29]. To date, about 140 archaeal viruses have been isolated, and most of these belong to the order Caudovirales, which is composed of three families of icosahedral tailed viruses [4-6, 19, 29]. In addition to these, spherical and linear, spindle-, bottle- and dropletshaped, and pleomorphic viruses are known to infect archaea [4, 6, 29].

Archaeal viruses have revealed deep evolutionary relationships between viruses infecting organisms from all three domains of life. Structural studies have shown that tailless icosahedral viruses infecting archaea, bacteria, or eukaryotes share a common ancestor [1, 9, 10, 12, 13, 33]. Furthermore, icosahedral tailed viruses infecting archaea and bacteria have recently been shown to have the same 
major capsid protein fold, pointing to a common origin [28]. Pleomorphic viruses infecting archaea and bacteria provide yet another example of viral relationships across domain barriers, as these viruses resemble each other at the virion level [16, 24-26]. The first isolate of pleomorphic, membrane-containing viruses infecting archaea, Halorubrum pleomorphic virus 1 (HRPV-1), was discovered in 2009, and since then, several other isolates have been characterized [3, 21, 24, 34]. Comprehensive studies of the pleomorphic archaeal viruses have been performed, but these viruses have remained unclassified [3, 18, 21, 24-26, $34,36]$. We have proposed to classify these viruses into a new viral family designated as Pleolipoviridae, and here, we summarize the available information on their virion components, genomic data and relatedness.

\section{Pleolipoviridae, a new family of eight archaeal pleomorphic viruses}

To date, eight pleomorphic membrane-containing viruses infecting halophilic archaea of the phylum Euryarchaeota have been discovered (Table 1). These viruses originate from globally distant locations, and five of them, Halorubrum pleomorphic viruses 1, 2, 3, and 6 (HRPV-1, HRPV2, HRPV-3, and HRPV-6) as well as Halogeometricum pleomorphic virus 1 (HGPV-1), have been isolated using a host strain originating from the same sample (Fig. 1). Haloarcula hispanica pleomorphic viruses 1 and 2 (HHPV1 and HHPV-2) and His2 have been isolated using a culture collection strain of Haloarcula hispanica [11, 21, 34]. These eight isolates share both genomic and structural features, showing that they are related. The distinguishing characteristics of this virus group are virion morphology and structural components, genome organization and gene synteny, and sequence similarity [7, 21, 24-26, 29, 34, 36]. When compared to the other known viruses, the only resemblance that haloarchaeal pleolipoviruses have is to pleomorphic viruses infecting bacterial mycoplasmas $[2,7$, 16, 24, 29]. However, only one of these phages, L2, has been classified (in the family Plasmaviridae) [19]. This isolate shares no detectable sequence similarity to the pleomorphic archaeal viruses. Consequently, we propose the creation of a new family, Pleolipoviridae, to classify the pleomorphic archaeal viruses. The name Pleolipoviridae originates from the Greek pleo for more or many and lipos for lipid as the members of the proposed family have pleomorphic virions that are composed of a proteinaceous lipid vesicle enclosing the genome (Fig. 2 and 3). The current members of the proposed family are divided into three genera (see below). The members are referred to as pleolipoviruses, and the model virus system HRPV-1 is the best characterized one.

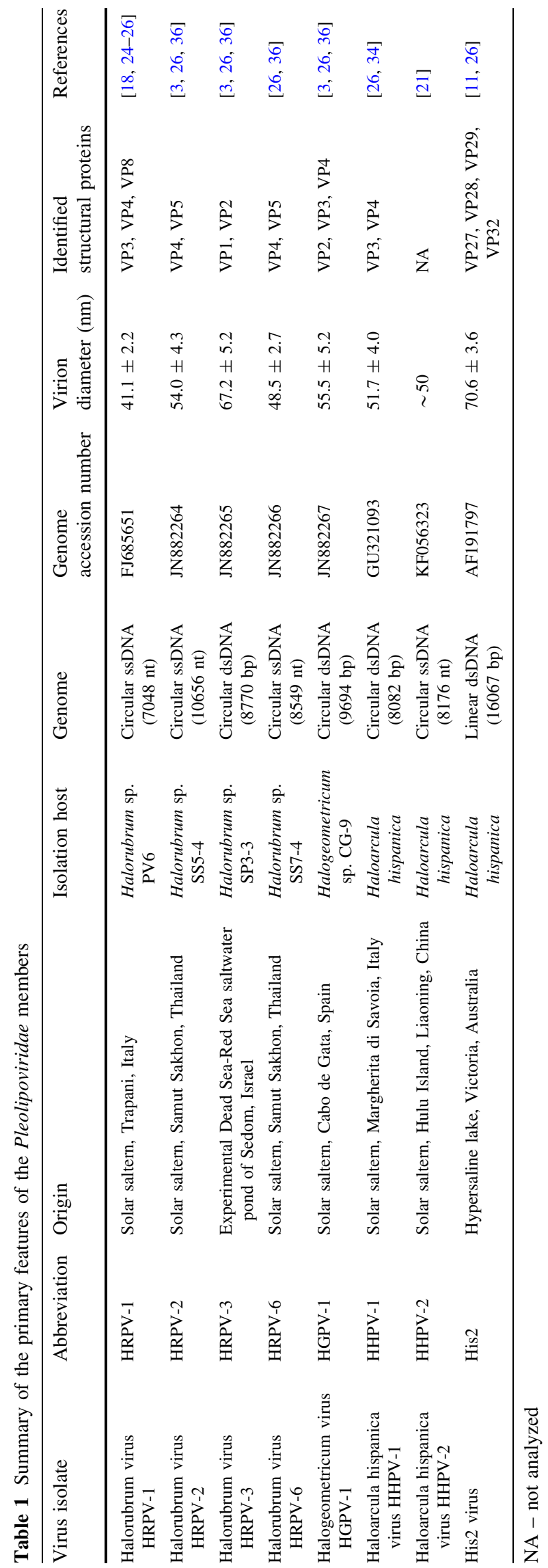




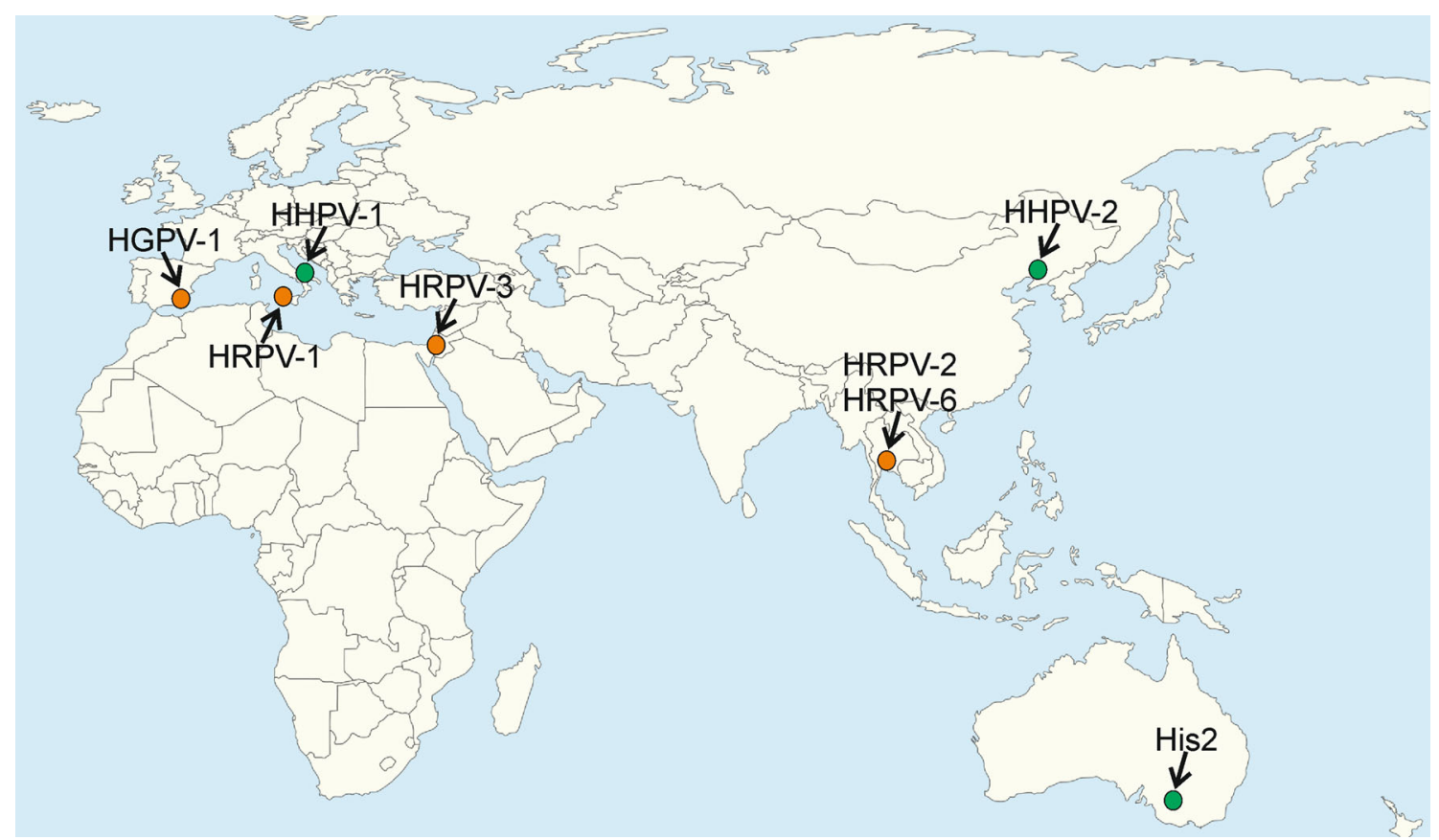

Fig. 1 Members of the proposed family Pleolipoviridae are globally distributed. Dots indicate the origin of virus isolates. Orange indicates that the virus was isolated using a host strain isolated from the same location, and green indicates that the virus was isolated using a culture collection strain of Haloarcula hispanica [3, 11, 21, 24, 26, 34]. Source of the map: Wikimedia Commons

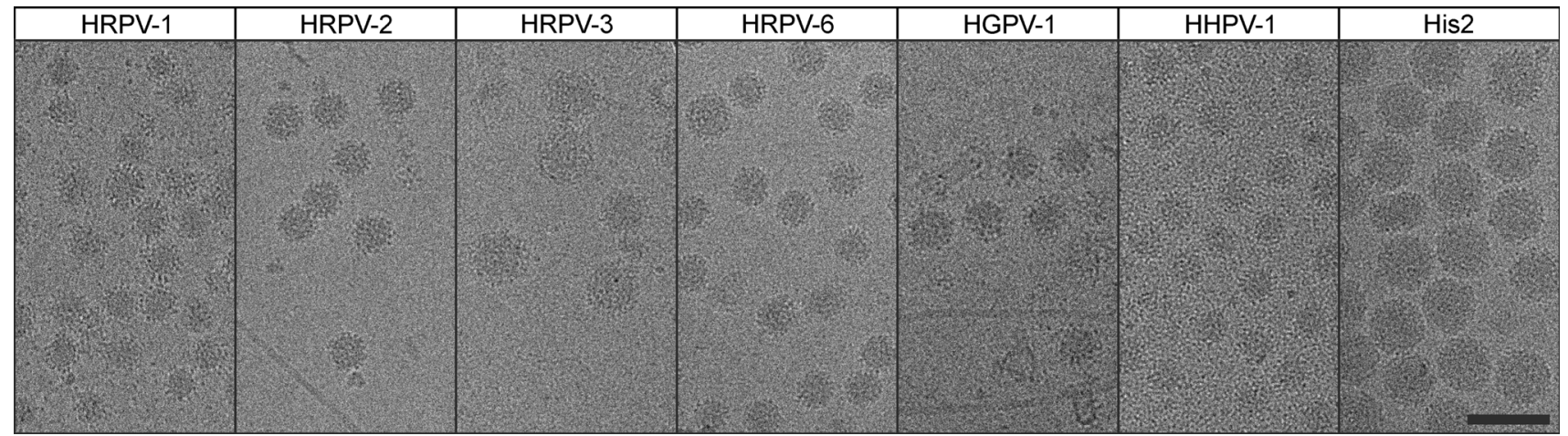

Fig. 2 Cryo-electron microscopy images of seven pleolipovirus isolates. Scale bar, $100 \mathrm{~nm}$. Reproduced from reference 26 with permission

His 2 virus is one of the members of the proposed family Pleolipoviridae. It has previously been suggested to be distantly related to the spindle-shaped virus His1 infecting Haloarcula hispanica [11]. Currently, His1 is classified as the type member of the floating genus Salterprovirus, and His2 has been listed as a virus that may be a member of the genus Salterprovirus [19]. However, His1 and His2 share no significant amino acid sequence similarity except for their putative DNA polymerases [11]. These proteinprimed family B DNA polymerases of His1 and His2 have been independently acquired from archaeal transposon-like elements [20]. Cryo-electron microscopy (cryo-EM) studies revealed that His2 is not spindle-shaped like His1, but rather spherical in shape (Fig. 2) [17, 26]. In addition, the
His2 virion has a canonical structural protein profile similar to those of the other isolates of the proposed family Pleolipoviridae (Fig. 3A), whereas the His1 virion protein pattern is unique $[26,27]$. The genome synteny and amino acid sequence similarity also suggest a relationship between His2 and the other pleolipoviruses [24, 34, 36]. Thus, we propose that His2 should be classified as a member of the proposed family Pleolipoviridae.

In addition to the pleolipoviruses described above, three more haloarchaeal pleomorphic viruses, Halorubrum pleomorphic viruses 7 and 8 (HRPV-7 and HRPV-8) and Haloarcula pleomorphic virus 2 (HAPV-2) have recently been isolated [5]. These isolates display characteristics of pleolipoviruses: pleomorphic virion morphotypes observed 


\section{A}

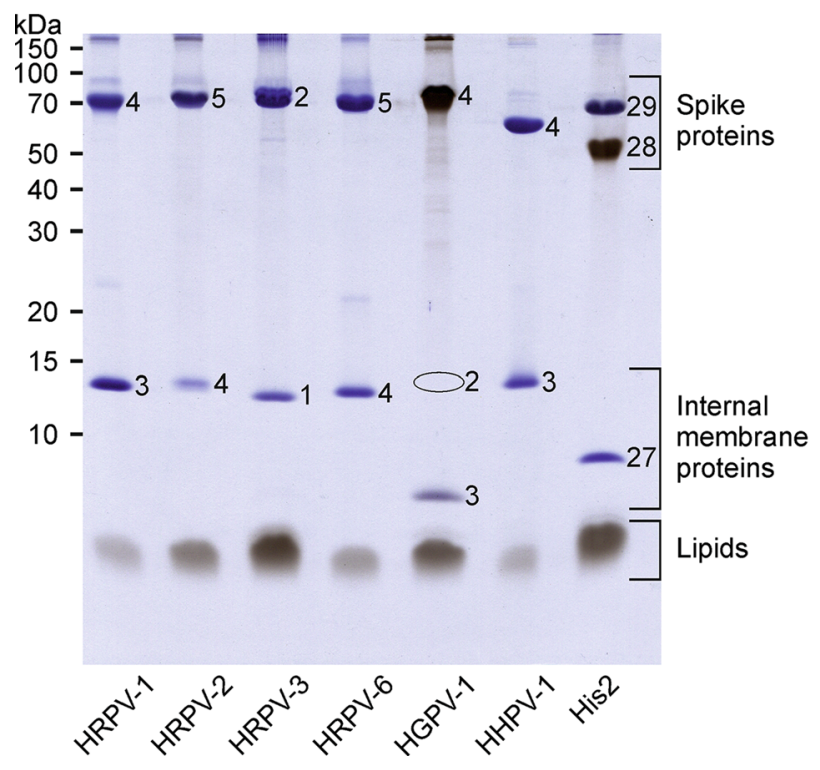

B
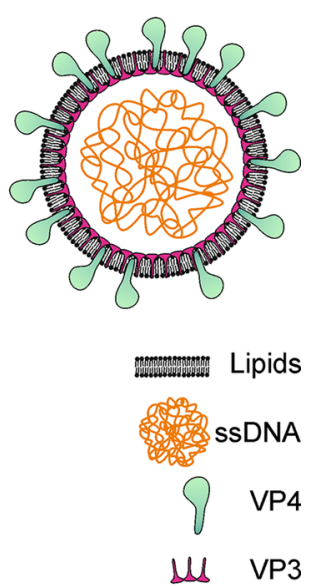

Fig. 3 Structural components of the seven pleolipoviruses. (A) Protein and lipid profile of the purified virions in a tricine-SDSpolyacrylamide gel stained with Coomassie blue and Sudan black B for proteins and lipids, respectively. Numbers on the left indicate the molecular masses of the markers. Numbers on the gel indicate the gene encoding the protein. The theoretical position of VP2 protein of
HGPV-1 is indicated by a circle. Reproduced from reference 26 with permission. Protein and lipid profiles are not available for HHPV-2. (B) Schematic representation of the HRPV-1 virion. HRPV-1 is the model virus of the proposed family Pleolipoviridae. Genomes of the pleolipoviruses can be either ssDNA or dsDNA, linear or circular

is the fusion of the virion envelope with the host cell membrane [24, 35].

\section{Pleomorphic appearance of pleolipoviruses}

Pleolipoviruses are sensitive to conditions of low salt concentration, confirming their halophilic nature [24-26]. Negative-stain transmission EM of the highly purified virions has suggested that the pleolipoviruses have a flexible virion structure that is not defined by a rigid protein capsid [21, 24-26, 34]. The pleomorphic appearance of the virions, which varies from spherical to elongated, does not resemble any of the previously described archaeal viruses $[30,32]$. To avoid possible artifacts caused by negative staining, the virion morphology of the pleolipoviruses has also been studied using cryo-EM and cryo-electron tomography (cryo-ET). The cryo-electron micrographs show roughly spherical particles with decorating spikes on the virion surface (Fig. 2) [26]. It has been observed that the dimensions of the individual viruses vary. The smallest of the viruses is HRPV-1 $(41.1 \pm 2.2 \mathrm{~nm})$, and the largest is His2 $(70.6 \pm 3.6 \mathrm{~nm})$ [26]. The pleomorphicity of the viruses is thus obvious in the range of sizes that each virus exhibits. In addition, cryo-ET of HRPV-1 has shown that there is an apparent lack of longitudinal order in the surface spikes, emphasizing the pleomorphicity [26]. 


\section{Virions of pleolipoviruses are simple and resemble membrane vesicles}

In addition to their morphology, pleolipoviruses have a highly similar, simple structural protein profile (Fig. 3A). Although a protein profile is not available for HHPV-2, the high similarity of all of its predicted genes to those of HHPV-1 suggests that the protein profiles of these two viruses are essentially the same. The virions of pleolipoviruses are composed of two major structural protein species [24-26, 34]. The smaller-sized protein contains predicted transmembrane domains, and the larger-sized one has a C-terminal membrane anchor preceded by a predicted coiled-coil domain. Quantitative biochemical dissociation analysis has shown that the larger-sized proteins of pleolipoviruses are anchored to the membrane and that the smaller ones are in the membrane, facing the particle interior, where the genome resides (Fig. 3B) [25, 26]. There are no nucleoproteins associated with the genome. Thus, the two major protein species have been designated as the spike protein (VP4-like protein according to the HRPV-1 nomenclature; VP for virion protein) and the internal membrane protein (VP3-like protein according to the HRPV-1 nomenclature). HHPV-1, HRPV-1, HRPV-2, HRPV-3, and HRPV-6 have one of each, His2 has two spike proteins and HGPV-1 has two internal membrane proteins (VP2 and VP3; Fig. 3A) [26]. The internal membrane protein VP27 of His2 shares amino-acid-level sequence similarity only with the HGPV-1 protein VP3 and is functionally a VP3-like protein [26]. At the amino acid sequence level, VP3-like proteins are rather conserved in all pleolipoviruses, except in His2.

Cryo-electron tomography has shown that HRPV-1 spikes formed of protein VP4 are randomly distributed on the virion surface. Furthermore, the HRPV-1 internal membrane protein VP3 is mostly embedded in the envelope and does not form an ordered protein capsid or a thick matrix-like layer on the inner surface of the membrane [26]. In HRPV-1, one minor structural protein, VP8, has been identified. HRPV-1 VP8, with its putative counterparts in other pleolipoviruses, is predicted to be an NTPase $[24,36]$.

Some of the pleolipoviruses have modifications in their spike proteins. HRPV-1 VP4 is glycosylated [18, 25], and the major $\mathrm{N}$-glycan is a pentasaccharide comprising glucose, glucuronic acid, mannose, sulphated glucuronic acid and a terminal 5-N-formyl-legionaminic acid residue [18]. This modification is involved in virus infectivity [18]. The spike proteins of His2 (VP28) and HGPV-1 (VP4) has been observed to be modified by unidentified lipid moieties [26].

Members of the proposed family Pleolipoviridae seem to acquire their lipid envelope from the host cell membrane, because the virions contain the same ratios of the major polar lipids as their host cells (the lipid profile is not available for HHPV-2) [24-26, 34]. Furthermore, it has been shown that the ratio of different lipids is the same in the viral and host membrane, indicating that the pleolipoviruses acquire their lipids unselectively from the host lipid pool $[25,34,38]$. Except for HGPV-1, the pleolipoviruses have three major phospholipids: phosphatidylglycerol (PG), phosphatidylglycerophosphate methyl ester (PGP-Me) and phosphatidylglycerosulfate (PGS) [24-26, 34]. The two major phospholipids of HGPV-1 and its host are PG and PGP-Me [26].

\section{The pleolipoviral genomes are either single- stranded or double-stranded DNA molecules}

All archaeal viruses characterized so far have a DNA genome, in contrast to known bacterial and eukaryotic viruses, which have either an RNA or DNA genome $[6,19$, 29]. Until 2009, the genomic landscape of the studied archaeal viruses was limited to double-stranded (ds) DNA genomes. HRPV-1 was the first archaeal virus to be described containing a single-stranded (ss) DNA genome [24]. Since the isolation of HRPV-1, four more ssDNA viruses infecting archaea have been described [21, 23, 36]. Three of them are members of the proposed family Pleolipoviridae.

The genomes of the eight pleolipoviruses discussed here have been sequenced (Table 1). The nucleotide sequence similarity of the genomes to other sequences in the databases is very limited if the other pleolipoviruses are excluded. The genomes show collinear gene organization (Fig. 4), but the genomes of HRPV-1, HRPV-2, HRPV-6 and HHPV-2 are ssDNA molecules, whereas HHPV-1 and His2 have dsDNA genomes [11, 21, 24, 34, 36]. HRPV-3 and HGPV-1 contain dsDNA genomes, but with stretches of ssDNA [36]. His2 has a linear genome, and the other virus isolates have circular ones. The length of the circular genomes varies from 7,048 nt (HRPV-1) to 10,656 nt (HRPV-2), and the linear His2 genome is $16,067 \mathrm{bp}$ in size (Table 1). The GC content of the genomes varies between $40 \%$ (His 2 ) and $64 \%$ (HRPV-2). At the nucleotide sequence level, the genomes show similarity $(60 \%$ or higher) only along very short stretches. Exceptions to this are the HRPV-2 and HRPV-6 genomes as well as HHPV-1 and HHPV-2 genomes, which show considerable nucleotide sequence similarity. The set of canonical core genes of the pleolipoviruses consists of genes encoding the internal membrane and spike protein and three conserved predicted downstream genes, one of which is predicted to encode an NTPase (Fig. 4) [11, 24, 34, 36]. 


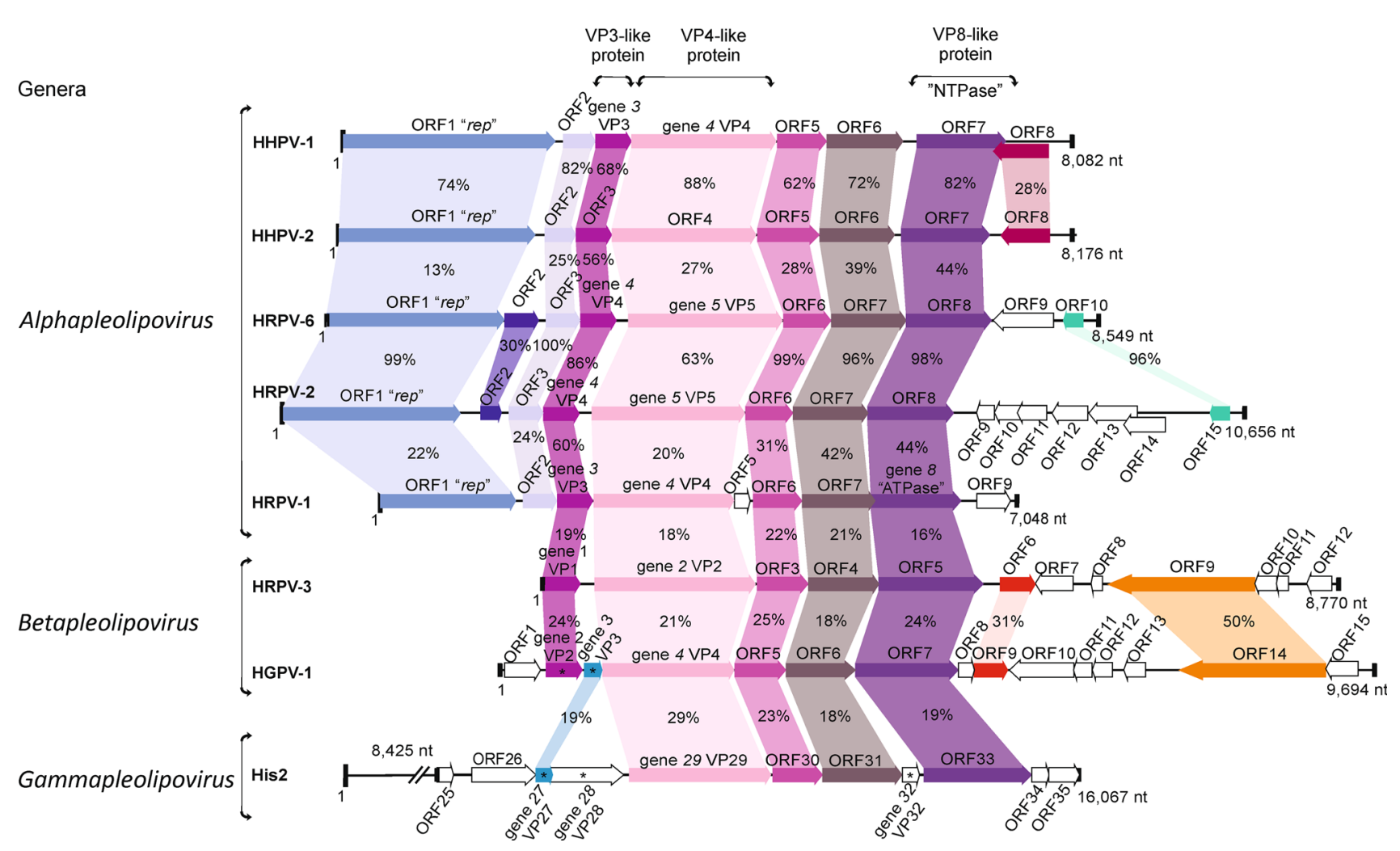

Fig. 4 A linear representation of the pleolipovirus genomes. The identities (\%) between the amino acid sequences of two predicted (or identified) gene products are indicated. Based on their genome

Among the proposed pleolipoviruses, the highest identity at the amino acid level can be found between the internal membrane VP3-like proteins (Fig. 4) [36]. One of the internal membrane proteins of HGPV-1 (VP3) shows similarity to the corresponding protein of His2 (VP27), and the other one (VP2) shows similarity to the internal membrane proteins of the other pleolipoviruses. In addition to the core genes, HRPV-1, HHPV-1, HHPV-2, HRPV-2 and HRPV-6 share a predicted gene encoding a putative rolling-circle replication initiation protein. The genomes of HRPV-3, HGPV-1 and His2 do not contain this putative gene but encode a protein homolog containing a C-terminal winged helix-turn-helix (wHTH) domain (HRPV-3 and HGPV-1) or a putative protein-primed family-B-type DNA polymerase (His2) [11, 36]. Thus, HRPV-1, HRPV-2, HRPV-6, HHPV-1 and HHPV-2 are proposed to use a rolling-circle replication mechanism $[24,34,36]$. The ends of the linear dsDNA genome of His2 contain inverted sequence repeats and terminal proteins and most likely replicate using protein priming, whereas the replication mechanisms of HRPV-3 and HGPV-1 remain unknown $[11,31,36]$.

A total of fourteen putative pleolipovirus-like proviruses have been identified in the genomes of haloarchaeal strains organization and the relatedness of their VP3-like proteins, the pleolipoviruses can be divided into three genera, which are indicated on the left

from the genera Haloarcula, Haloferax, Halomicrobium, Halopiger, Halorhabdus, Natrialba, Haloterrigena and Natronomonas [15, 24, 34-36]. Haloferax plasmid pHK2 and Halorubrum plasmid pHRDV1 show gene synteny and significant amino acid sequence similarity to the pleolipovirus genomes. Thus, these plasmids are most likely proviruses related to the pleolipoviruses [14, 34]. Also, a metagenome from a hypersaline lake contained a sequence similar to those of the pleolipoviruses [37].

\section{Archaeal pleolipoviruses and bacterial mycoplasmaviruses}

Pleolipovirus-like morphology has also been observed among bacterial mycoplasmaviruses. The pleomorphic, enveloped phages L2 and L172, which infect Acholeplasma laidlawii cells, have circular dsDNA and ssDNA genomes, respectively $[16,22]$. However, there is no detectable DNA homology between these viruses [16]. Both L2 and L172 acquire their lipids unselectively from the host cell membrane, as do pleolipoviruses [2]. Notably, the protein profile of L172 is very similar to that of the pleolipoviruses, as there are two major protein 
Table 2 Taxonomic structure of the proposed family Pleolipoviridae

\begin{tabular}{lll}
\hline Genus & Species & Representive isolate $^{\mathrm{a}}$ \\
\hline Alphapleolipovirus & Halorubrum virus HRPV-1 (type species) & Halorubrum pleomorphic virus 1 (HRPV-1) \\
& Halorubrum virus HRPV-2 & Halorubrum pleomorphic virus 2 (HRPV-2) \\
& Halorubrum virus HRPV-6 & Halorubrum pleomorphic virus 6 (HRPV-6) \\
& Haloarcula virus HHPV-1 & Haloarcula hispanica pleomorphic virus 1 (HHPV-1) \\
& Haloarcula virus HHPV-2 & Haloarcula hispanica pleomorphic virus 1 (HHPV-2) \\
Betapleolipovirus & Halorubrum virus HRPV-3 (type species) & Halorubrum pleomorphic virus 3 (HRPV-3) \\
Gammapleolipovirus & Halogeometricum virus HGPV-1 & Halogeometricum pleomorphic virus 1 (HGPV-1) \\
\hline
\end{tabular}

a The abbreviation of the virus name is given in parentheses

components, and their estimated masses are close to those of the major structural proteins of the pleolipoviruses [16, $24,26]$. As there is no sequence data available for L172, its classification is currently unclear. The protein profile of L2 differs from that of L172 and the pleolipoviruses [16, 26], and L2 shows no sequence similarity to the pleolipoviruses. Thus, the proposal of a new family for archaeal pleolipoviruses is in line with the current classification of L2 into the family Plasmaviridae.

\section{Taxonomic structure of the proposed family Pleolipoviridae}

We propose the following genus and species demarcation criteria for the family Pleolipoviridae (Table 2): (i) Alphapleolipovirus: A sequence comparison of viruses belonging to different species shows low sequence similarity over the whole genomic nucleotide sequence, but their genomes are collinear. All members encode a putative rolling-circle replication initiation protein. (ii) Betapleolipovirus: A sequence comparison of viruses belonging to different species shows very little sequence similarity, but their genomes are collinear. Members encode a conserved haloarchaeal protein containing a winged-helix DNAbinding domain. (iii) Gammapleolipovirus: This proposed genus currently includes only one member, Haloarcula virus His2, which has a gene encoding a putative proteinprimed family-B-type DNA polymerase (Table 2).

Among the canonical pleolipoviral gene products, the VP3-like internal membrane protein shows the highest similarity at the amino acid sequence level [36]. The relatedness of the VP3-like proteins can also be used to divide the current members of the Pleolipoviridae into the three genera in the same way as with the above-proposed criteria based on gene content. The relatedness of $\mathrm{Al}$ phapleolipovirus members, which have either an ssDNA or dsDNA genome, can be further verified on the basis of
VP3-like protein relatedness. In this case, the genome type is not an adequate criterion.

In conclusion, our recent data show that the eight sequenced pleolipoviruses infecting halophilic archaea share a conserved vesicle-like virion architecture. Based on this canonical virion architecture, pleolipoviruses differ from other known enveloped viruses, as there is no nucleoprotein or matrix protein typical of such viruses. Despite the different genome types, the pleolipoviruses share genome synteny. Accordingly, we propose a new viral family for these viruses, Pleolipoviridae. Subdivision of the family into three genera, Alphapleolipovirus, Betapleolipovirus, and Gammapleolipovirus, is proposed. Traditionally, the genome type has been an important criterion in virus classification $[8,19]$. Thus, the proposed family Pleolipoviridae challenges this view by having both ssDNA and dsDNA viruses as well as both linear and circular genomes. This is most likely due to the replication strategies used resulting in different types of DNA molecules being encapsidated into a virion. We hope that further studies and comparisons will show whether the proposed archaeal virus family Pleolipoviridae and the phage family Plasmaviridae could form an order, for which we propose here the name Pleolipovirales.

Acknowledgments This work was supported by an Academy of Finland Postdoctoral Researcher funding grant (1274748; M.K.P), and Academy Professor funding grants (283072 and 255342; D.H.B.). We thank the Academy of Finland (grants 271413 and 272853) and the University of Helsinki for the support to the EU ESFRI Instruct Centre for Virus Production (ICVIR). We also acknowledge the Czech Science Foundation (grant P302/11/1940) and funding from RVO61388971 for support.

We acknowledge all authors in the major original papers of pleolipoviruses (Table 1) for their valuable contribution. In alphabetical order: Aitio O, Atanasova NS, Bath C, Butcher SJ, Cukalac T, Domanska A, Dyall-Smith ML, Eichler J, Guan Z, Helin J, Helm M, Kalkkinen N, Kandiba L, Kellner S, Kukkaro P, Laurinavicius S, Li M, Liljeroos L, Manole V, Oren A, Paulin L, Permi P, Porter K, Somerharju P, Sund J, Wang R, Xiang H and Zhao D. 


\section{References}

1. Abrescia NG, Bamford DH, Grimes JM, Stuart DI (2012) Structure unifies the viral universe. Annu Rev Biochem $81: 795-822$

2. Al-Shammari AJN, Smith PF (1981) Lipid composition of two mycoplasmaviruses, MV-Lg-L172 and MVL2. J Gen Virol $54: 455-458$

3. Atanasova NS, Roine E, Oren A, Bamford DH, Oksanen HM (2012) Global network of specific virus-host interactions in hypersaline environments. Environ Microbiol 14:426-440

4. Atanasova NS, Bamford DH, Oksanen HM (2015) Haloarchaeal virus morphotypes. Biochimie. doi:10.1016/j.biochi.2015.07.002

5. Atanasova NS, Demina TA, Buivydas A, Bamford DH, Oksanen HM (2015) Archaeal viruses multiply: temporal screening in a solar saltern. Viruses 7:1902-1926

6. Atanasova NS, Oksanen HM, Bamford DH (2015) Haloviruses of archaea, bacteria and eukaryotes. Curr Opin Microbiol 25:40-48

7. Atanasova NS, Sencilo A, Pietilä MK, Roine E, Oksanen HM, Bamford DH (2015) Comparison of lipid-containing bacterial and archaeal viruses. Adv Virus Res 92:1-61

8. Baltimore D (1971) Expression of animal virus genomes. Bacteriol Rev 35:235-241

9. Bamford DH, Burnett RM, Stuart DI (2002) Evolution of viral structure. Theor Popul Biol 61:461-470

10. Bamford DH (2003) Do viruses form lineages across different domains of life? Res Microbiol 154:231-236

11. Bath C, Cukalac T, Porter K, Dyall-Smith ML (2006) His1 and His2 are distantly related, spindle-shaped haloviruses belonging to the novel virus group, Salterprovirus. Virology 350:228-239

12. Benson SD, Bamford JK, Bamford DH, Burnett RM (1999) Viral evolution revealed by bacteriophage PRD1 and human adenovirus coat protein structures. Cell 98:825-833

13. Benson SD, Bamford JK, Bamford DH, Burnett RM (2004) Does common architecture reveal a viral lineage spanning all three domains of life? Mol Cell 16:673-685

14. Chen S, Wang C, Xu JP, Yang ZL (2014) Molecular characterization of pHRDV1, a new virus-like mobile genetic element closely related to pleomorphic viruses in haloarchaea. Extremophiles 18:195-206

15. Dyall-Smith ML, Pfeiffer F, Klee K, Palm P, Gross K, Schuster SC, Rampp M, Oesterhelt D (2011) Haloquadratum walsbyi: limited diversity in a global pond. PLoS One 6:e20968

16. Dybvig K, Nowak JA, Sladek TL, Maniloff J (1985) Identification of an enveloped phage, mycoplasma virus L172, that contains a 14-kilobase single-stranded DNA genome. J Virol 53:384-390

17. Hong C, Pietilä MK, Fu CJ, Schmid MF, Bamford DH, Chiu W (2015) Lemon-shaped halo archaeal virus His1 with uniform tail but variable capsid structure. Proc Natl Acad Sci USA 112:2449-2454

18. Kandiba L, Aitio O, Helin J, Guan Z, Permi P, Bamford DH, Eichler J, Roine E (2012) Diversity in prokaryotic glycosylation: an archaeal-derived N-linked glycan contains legionaminic acid. Mol Microbiol 84:578-593

19. King AMQ, Adams MJ, Carstens EB, Lefkowitz EJ (2012) Virus taxonomy: ninth report of the International Committee on Taxonomy of Viruses. Elsevier Academic Press, London

20. Krupovic M, Makarova KS, Forterre P, Prangishvili D, Koonin EV (2014) Casposons: a new superfamily of self-synthesizing DNA transposons at the origin of prokaryotic CRISPR-Cas immunity. BMC Biol 12:36
21. Li M, Wang R, Zhao D, Xiang H (2014) Adaptation of the Haloarcula hispanica CRISPR-Cas system to a purified virus strictly requires a priming process. Nucleic Acids Res 42:2483-2492

22. Maniloff J, Kampo GJ, Dascher CC (1994) Sequence analysis of a unique temperature phage: mycoplasma virus L2. Gene 141:1-8

23. Mochizuki T, Krupovic M, Pehau-Arnaudet G, Sako Y, Forterre P, Prangishvili D (2012) Archaeal virus with exceptional virion architecture and the largest single-stranded DNA genome. Proc Natl Acad Sci USA 109:13386-13391

24. Pietilä MK, Roine E, Paulin L, Kalkkinen N, Bamford DH (2009) An ssDNA virus infecting archaea: a new lineage of viruses with a membrane envelope. Mol Microbiol 72:307-319

25. Pietilä MK, Laurinavičius S, Sund J, Roine E, Bamford DH (2010) The single-stranded DNA genome of novel archaeal virus Halorubrum pleomorphic virus 1 is enclosed in the envelope decorated with glycoprotein spikes. J Virol 84:788-798

26. Pietilä MK, Atanasova NS, Manole V, Liljeroos L, Butcher SJ, Oksanen HM, Bamford DH (2012) Virion architecture unifies globally distributed pleolipoviruses infecting halophilic archaea. J Virol 86:5067-5079

27. Pietilä MK, Atanasova NS, Oksanen HM, Bamford DH (2013) Modified coat protein forms the flexible spindle-shaped virion of haloarchaeal virus His1. Environ Microbiol 15:1674-1686

28. Pietilä MK, Laurinmäki P, Russell DA, Ko CC, Jacobs-Sera D, Hendrix RW, Bamford DH, Butcher SJ (2013) Structure of the archaeal head-tailed virus HSTV-1 completes the HK97 fold story. Proc Natl Acad Sci USA 110:10604-10609

29. Pietilä MK, Demina TA, Atanasova NS, Oksanen HM, Bamford DH (2014) Archaeal viruses and bacteriophages: comparisons and contrasts. Trends Microbiol 22:334-344

30. Pina M, Bize A, Forterre P, Prangishvili D (2011) The archeoviruses. FEMS Microbiol Rev 35:1035-1054

31. Porter K, Dyall-Smith ML (2008) Transfection of haloarchaea by the DNAs of spindle and round haloviruses and the use of transposon mutagenesis to identify non-essential regions. Mol Microbiol 70:1236-1245

32. Prangishvili D, Forterre P, Garrett RA (2006) Viruses of the Archaea: a unifying view. Nat Rev Microbiol 4:837-848

33. Rice G, Tang L, Stedman K, Roberto F, Spuhler J, Gillitzer E, Johnson JE, Douglas T, Young M (2004) The structure of a thermophilic archaeal virus shows a double-stranded DNA viral capsid type that spans all domains of life. Proc Natl Acad Sci USA 101:7716-7720

34. Roine E, Kukkaro P, Paulin L, Laurinavičius S, Domanska A, Somerharju P, Bamford DH (2010) New, closely related haloarchaeal viral elements with different nucleic acid types. J Virol 84:3682-3689

35. Roine E, Oksanen HM (2011) Viruses from the hypersaline environment. In: Ventosa A, Oren A, Ma Y (eds) Halophiles and hypersaline environments: current research and future trends. Springer, Berlin, pp 153-172

36. Senčilo A, Paulin L, Kellner S, Helm M, Roine E (2012) Related haloarchaeal pleomorphic viruses contain different genome types. Nucleic Acids Res 40:5523-5534

37. Sime-Ngando T, Lucas S, Robin A, Tucker KP, Colombet J, Bettarel Y, Desmond E, Gribaldo S, Forterre P, Breitbart M, Prangishvili D (2011) Diversity of virus-host systems in hypersaline Lake Retba, Senegal. Environ Microbiol 13:1956-1972

38. Vitale R, Roine E, Bamford DH, Corcelli A (2013) Lipid fingerprints of intact viruses by MALDI-TOF/mass spectrometry. Biochim Biophys Acta 1831:872-879 